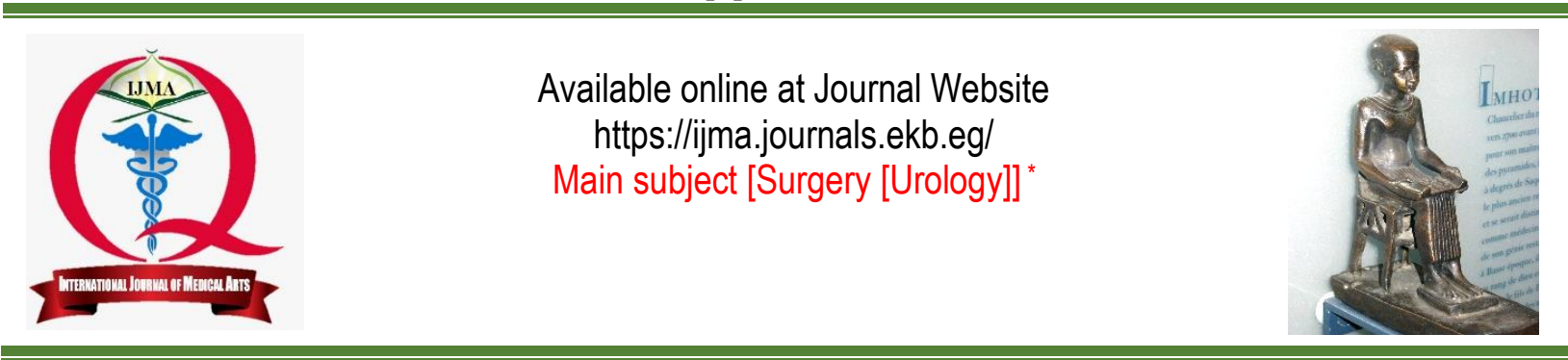

Case Report

\title{
Robotic Assisted Laparoscopic Partial Cystectomy and En Bloc Resection of Urachus and Umbilicus for Rare Case of Mucin Producing Urachal Adenocarcinoma: A Case Report
}

\section{Nabeel Joda Kuwaijo[1]; Stephanie Curran[2]; Paddy O'Malley[1]}

Department of Urology, The Galway Clinic, Royal College of Surgeons, Ireland ${ }^{[1]}$

Department of Pathology, The Galway Clinic, Royal College of Surgeons, Ireland [2]

Corresponding author: Paddy O'Malley

Email: paddy.omalley@galwayclinic.com

Received at: March 31, 2020; Revised at: June 26, 2020; Accepted at: July 4, 2020; Available online at: July 4, 2020

\section{DOI: $10.21608 / \mathrm{ijma} .2020 .25136 .1114$}

\section{ABSTRACT}

Background: Urachal carcinoma accounts for $<1 \%$ of all bladder cancers, $90 \%$ are adenocarcinomas, accounting for $10 \%$ of the bladders' adenocarcinomas. For its rarity, there is no consensus about the nomenclature, staging, diagnosis and treatment.

Case report: We described a 42 years old male, who complained of post-voiding mucinous discharge for a long period [10 years]. after extensive clinical evaluation and radiologic investigation and cystoscopy, the diagnosis of an early stage urachal adenocarcinoma had been confirmed. It had been completely excised with no metastasis. The surgical resection of the tumour done en-bloc through laparoscopy, roboticassisted surgery. No chemotherapy nor radiotherapy had been indicated, the patient has been submitted to close follow-up, with our hoping look for recurrence of the disease. Excised specimen had been further submitted to histopathological and immunostaining examination. Histopathological grading had been done according to most recent staging systems [it confirmed the early staging mucinous adenocarcinoma of urachal origin].

Conclusion: Here we described our experience with a rare case report of mucinous cystadenoma of urachal origin, robot-assisted laparoscopic surgery and early stage of cancer permits partial cystectomy, good outcome with no need for chemical or radiotherapy.

Keywords: Adenocarcinoma; Urachus; Partial Cystecomy; Robotic; Mucin

This is an open access article under the Creative Commons license [CC BY] [https://creativecommons.org/licenses/by/2.0/]

Please cite this article as: Kuwaijo NJ, Curran S, O'Malley P. Robotic Assisted Laparoscopic Partial Cystectomy and En Bloc Resection of Urachus and Umbilicus for Rare Case of Mucin Producing Urachal Adenocarcinoma: A Case Report. IJMA 2020; 2[3]: 554-558.

* Main subject and any subcategories have been classified according to research topic. 


\section{INTRODUCTION}

The urachus is a tube-like structure that connects the allantois to the urinary bladder in the embryonic development, shrinking after the third trimester of pregnancy to form a closed fibromuscular canal between the umbilicus and urinary bladder dome, Urachal ruminant can persist in approximately $32 \%$ of adults ${ }^{[1]}$, urachal carcinoma accounts for $<1 \%$ of all bladder cancers $[2,3], 90 \%$ are adenocarcinomas ${ }^{[4]}$, accounting for $10 \%$ of the bladders' adenocarcinoma[5]. Non-glandular neoplasms can be urothelial, squamous cells, neuroendocrine, and mixed type[6].

For its rarity, up to date, there is no consensus about the nomenclature, staging, diagnosis and treatment.

Generally, adenocarcinoma of the bladderprimary signet ring cell carcinoma- is a very rare tumor that occurs around less than $1 \%$ of all bladder carcinomas neoplasms ${ }^{[7]}$. Signet ring cell carcinoma can be of urachal origin and directly extend into the bladder. These tumours generally present as highgrade, high-stage tumours and have a uniformly poor prognosis ${ }^{[8]}$.

The primary treatment is radical cystectomy; however, in the majority of cases there are regional or distant metastases at the time of presentation [needs more extensive surgical intervention], and the mean survival time is less than 20 months ${ }^{[10]}$.

Here we report a case of urachal adenocarcinoma, that treated by robotic- assisted laparoscopic partial cystectomy and en bloc resection of urachus and umbilicus

\section{CASE REPORT}

We described a fit and healthy 42-years-old male, presented with a complaint of post-micturition mucinous discharge. His complaint extended for the last 10 years, with no hematuria or any associated lower urinary tract symptoms. His examination was unremarkable, and an abdominal computed tomographic scan revealing no abnormality out of tiny [ $3 \mathrm{~mm}]$ bilateral non-obstructing renal calculi. For persistent bothersome urethral discharge, the patient underwent magnetic resonance imaging [MRI], pelvic rigid cystoscopy [Figures 1 and 2] and examination under general anaesthesia. These examinations revealed a small orifice at the dome of the bladder with no mucosal erosion. 50/50 contrast had been injected to assess the extent and communication of this finding and it was blindly ended. No biopsy taken at this stage the tests confirmed communicating urachal ruminant. Based on these, a multidisciplinary decision was made to operate.

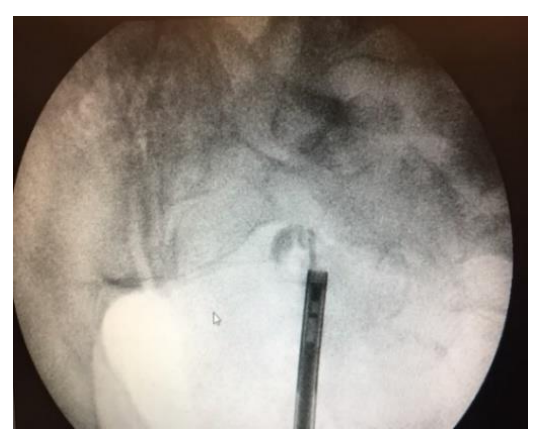

Figure [1]: Cystoscopy assessment

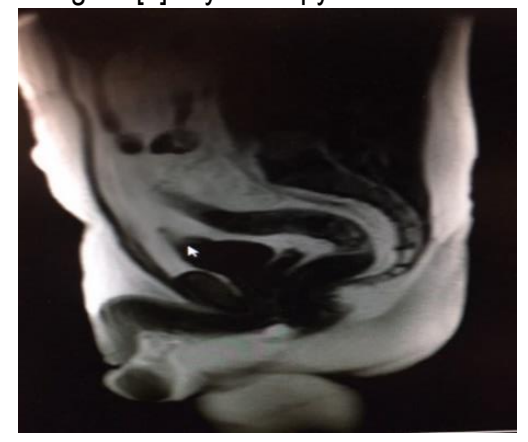

Figure [2]: MRI assessment

Our surgical approach had been adopted from Robotic Assisted Radical Cystoprostatectomy[11]. We performed flexible cystoscopy for localisation using trans-illumination. Umbilicus, subcutaneous tissue, part of rectus muscle \& its sheath, and the bladder dome, were removed En-bloc.

The tumour had been completely excised and it had been confirmed in the mmultidisciplinary teams [MDT] meeting. Specimens had been prepared for both histopathological and immunostaining studies.

A written consent has been signed by the patient to release information for this study.

\section{Gross description:}

Irregular portion of yellowish-brown tissue, overall $200 \times 165 x$ up to $20 \mathrm{~mm}$. The specimen includes umbilicus with surrounding skin with long suture, underlying fatty, firm tissue including perivesical fat, segment of the bladder wall marked with short suture attached as seen in the photograph [figure 3] 


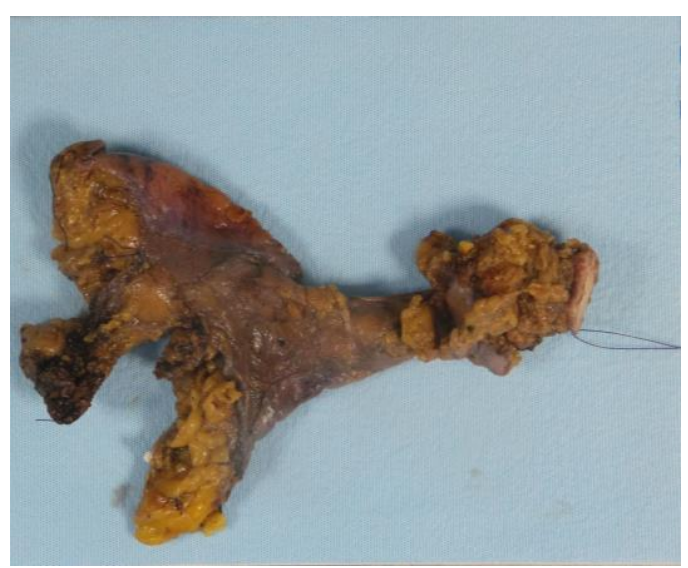

Figure [3]: Macroscopic aspects: superior view

\section{Microscopy:}

The specimens were fixed in formalin embedded in paraffin and were routinely stained with avid biotinperoxidase; its histology was reported as urachal adenocarcinoma of mucinous type as seen in figures [4-7].

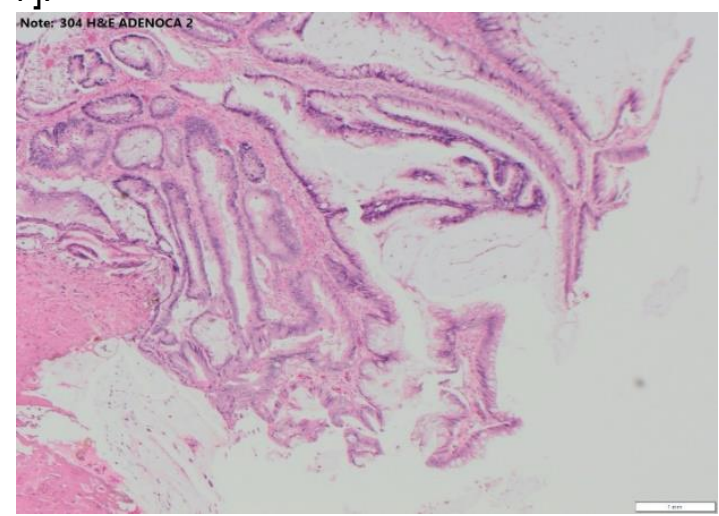

Figure [4] Urachus adenocarcinoma

The histology confirms presence of a urachal remnant. This is lined partially by transitional type urothelium and partially by glandular epithelium. The glandular epithelium has undergone neoplastic transformation producing a gland forming neoplasm that shows focal papillary glandular architecture protruding into the lumen of the urachal remnant but, elsewhere shows infiltrative growth i.e. adenocarcinoma which focally invades into the muscle coat of the urachus. In one section shows invasive tumour within the muscle layer of the urachus protruding through the wall, this focus measured $3.7 \mathrm{~mm}$.

Histologic features of the tumour were: [a] urothelium surface; [b] glandular differentiation; [c] areas of lesser differentiation. The Immunostains CDX2 positive staining in the tumor; $\beta$-catenin negative nuclear staining in the majority of the tumour. There was no inflammatory, low cellularity neither sclerotic stroma nor micro-vascular invasion [desmoplastic reaction]. Both cytokeratin 20 [CK20] and CDX2 showed expression and remained negative staining in the overlying urothelium.

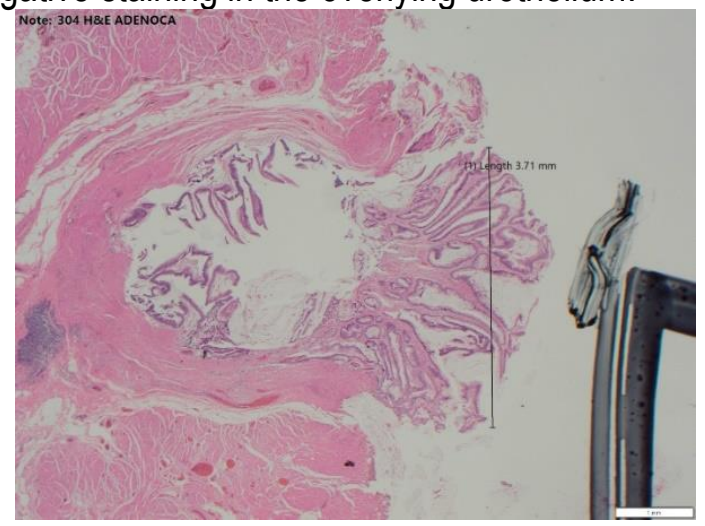

Figure [5]: Another image reflected adenocarcinoma

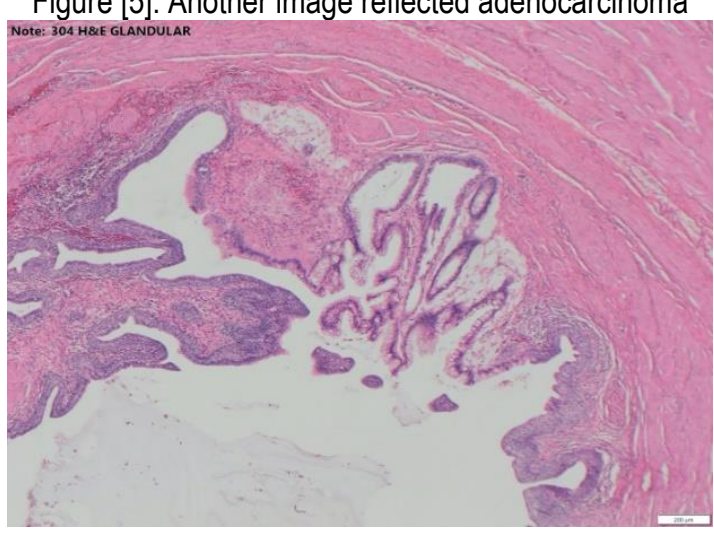

Figure [6]: Glandular elements of adenocarcinoma

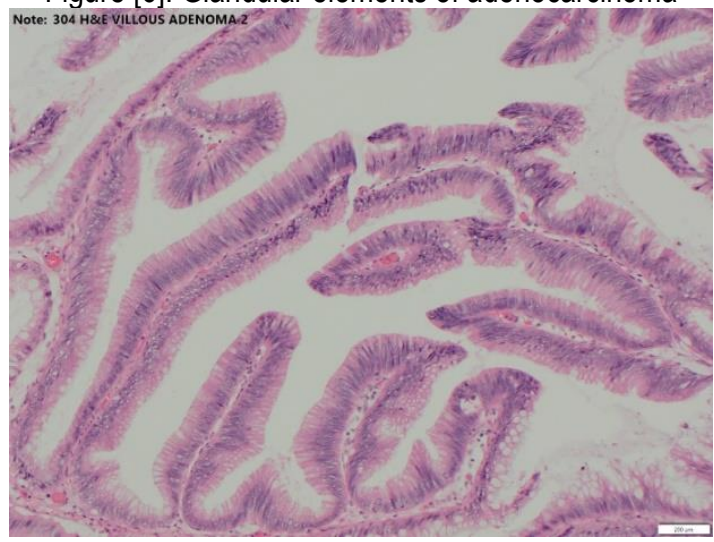

Figure [7]: Villous adenocarcinoma

\section{DISCUSSION}

Ural carcinoma is a rare clinical entity and had three characteristics, late presentation of symptoms, had early local invasion and usually had distal metastasis. All these criteria are associated in poor prognosis[12]. The first case reported in 1863 by Hue and Jacquin, subsequently Wheeler and Hill designed the diagnostic criteria for the urachus carcinoma in the 1950s, these criteria were modified, and they aren't without controversy, although most uro-oncologists agreeing both Mostofi et al. [13] and 
The Sheldon et al. ${ }^{[14]}$ criteria. However, these criteria had been criticised by previous studies[15-16]. For example, a deviation from the first two criteria, i.e., 1. tumor in the dome of the bladder, and 2. absence of cystitis cystica and cystitis glandularis, does not exclude the diagnosis of urachus cancer. Actually, urachal remnants have been reported in the midline or vertex in $54 \%$ of cases while they have been recognized in the anterior wall among $2 \%$ [17]

Most recently, the WHO blue book[18] for the diagnosis of urachal adenocarcinoma used new set of criteria from Gopalan et al.[19] which are: 1] the tumor located in bladder dome and/or anterior wall, 2] epicenter of carcinoma in the bladder wall, 3] absence of widespread cystitis cystica and/or cystitis glandularis beyond the dome and anterior wall, and 4] absence of a known primary tumour elsewhere.

The tumor reported in the current case had been located in the lumen of the urachal remnant which focally invades into the muscle coat of the urachus, with no other primary tumour fulfilling the WHO criteria for the diagnosis of urachal adenocarcinoma. It was a mucin secreting glandular adenocarcinoma of enteric type. The most commonly accommodated histological subtyping of urachal adenocarcinoma is enteric, mucinous, signet ring cell, and mixed type or not otherwise specified.

In our case Immuno-histochemistry: expressed CK20 and CDX2 was negative for both CK7 and $34 \beta$ E12 and $\beta$-catenin showed focal nuclear staining. These Immunohistochemical results support the diagnosis of urachal origin. $60 \%$ of the urachal adenocarcinoma are positive for CK20 and CDX2, CK7, and focally for $34 \beta \mathrm{E} 12$ in $66 \%$; nuclear staining with $6 \% \beta$-catenin ${ }^{[1]}$ which expressed more in colonic adenocarcinomas and also associated with no expression of CK7.

The differential diagnosis of urachal adenocarcinomas is adenocarcinomas either primary vesical or secondary colonic tumour, the treating urologist should exclude vesical, colonic origin, of the urachal tumour for that, immunostaining is very important[20].

As urachal adenocarcinomas being diagnosed in advance stages, they are associated with a poor prognosis with 50\% 5-year survival [2,3,5]. Comparing similar stages of Urachal adeno-carcinoma to bladder carcinoma, urachal carcinoma has a better survival rate[9]. Furthermore, there are some independent predictor factors, considered by some studies to influence the outcome[2], that are tumor spread outside of bladder to adjacent organs and abdominal wall, the surgical margin or local invasion of the tumour and distant metastases.

Our case was staged by Sheldon system ${ }^{[14]}$ as stage II and stage I by Mayo system ${ }^{[12]}$. This organ confined and completely excised disease, with no metastasis, will undergo a clinical and imaging follow-up to assess for disease recurrence. The recommended treatment for nonmetastatic cases is surgery. Partial or radical cystectomy has similar oncologic results. En bloc resection with complete removal of urachal remnant and the umbilicus should be the surgery performed for prolonged survival[4]; however, no chemotherapeutic regimen or Radiotherapy performed in our case because of the negative surgical margin.

\section{Conclusion}

In summary, we present a case of early stage urachal adenocarcinoma, which was completely excised with no metastasis. The surgical resection of the tumour done en-bloc, no chemotherapy nor radiotherapy was indicated, the patient will undergo close follow-up hoping looking for recurrence of the disease.

In the review we adapted from Gopalan et al. [19] published in the 2016 WHO blue book, and we used the Sheldon and Mayo systems for staging as they are most accepted ones. We found in few studies that urothelial carcinoma of same stage as urachal adenocarcinoma, has worse prognosis.

\section{Financial and Non-Financial Relationships and Activities of Interest}

None

\section{REFERENCES}

1. Wang D, Sule N. Mucinous Cystadenoma of the Urachus and Review of Current Classification of Urachal Mucinous Cystic Neoplasms. Arch Pathol Lab Med. 2019 Feb; 143[2]: 258-263. [DOI: 10.5858/arpa.2017-0319-RS].

2. Bruins HM, Visser $O$, Ploeg $M$, Hulsbergen-van de Kaa CA, Kiemeney LA, Witjes JA. The clinical epidemiology of urachal carcinoma: results of a large, population-based study. J Urol. 2012 Oct;188[4]:1102-7. [DOI: 10.1016/j.juro. 2012.06.020].

3. Pinthus JH, Haddad R, Trachtenberg J, Holowaty E, Bowler J, Herzenberg AM, Jewett M, Fleshner NE. 
Population based survival data on urachal tumors. J Urol. 2006 Jun;175[6]:2042-7. [DOI: 10.1016/S0022-5347[06] 00263-1].

4. Szarvas T, Módos O, Niedworok C, Reis H, Szendröi A, Szász MA, Nyirády P. Clinical, prognostic, and therapeutic aspects of urachal carcinoma-A comprehensive review with meta-analysis of 1,010 cases. Urol Oncol. 2016 Sep;34[9]:388-98. [DOI: 10.1016/j.urolonc.2016.04.012].

5. Wright JL, Porter MP, Li Cl, Lange PH, Lin DW. Differences in survival among patients with urachal and non-urachal adenocarcinomas of the bladder. Cancer. 2006 Aug 15;107[4]:721-8. [DOI: 10.1002/cncr.22059].

6. Humphrey PA, Moch H, Cubilla AL, Ulbright TM, Reuter VE. The 2016 WHO Classification of Tumours of the Urinary System and Male Genital Organs-Part B: Prostate and Bladder Tumours. Eur Urol. 2016 Jul;70[1]:106-119. [DOI: 10.1016/j.eururo.2016.02.028].

7. Hong SH, Kim JC, Hwang TK. Laparoscopic partial cystectomy with en bloc resection of the urachus for urachal adenocarcinoma. Int J Urol. 2007 Oct;14[10]:963-5. [DOI: 10.1111/j.1442-2042.2007. 01855.x].

8. Aggarwal A, Agarwal S, Pandey S, Sankhwar S. Urachal adenocarcinoma. BMJ Case Rep. 2018 Sep 15;2018: bcr2018226207. [DOI: 10.1136/bcr-2018-226207].

9. Claps M, Stellato M, Zattarin E, Mennitto A, Sepe P, Guadalupi V, et al. Current Understanding of Urachal Adenocarcinoma and Management Strategy. Curr Oncol Rep. 2020 Jan 27;22[1]:9. [DOI: 10.1007/s11912-0200878-z].

10. Yaegashi H, Kadomoto S, Naito R, Makino T, Iwamoto $\mathrm{H}$, Nohara $\mathrm{T}$, et al. Metastatic urachal cancer treated effectively with gemcitabine/cisplatin combination chemotherapy and radiotherapy: A case report. Mol Clin Oncol. 2019 Aug;11[2]:139-142. [DOI: 10.3892/mco. 2019. 1865].

11. Pruthi RS, Wallen EM. Robotic assisted laparoscopic radical cystoprostatectomy: operative and pathological outcomes. J Urol. 2007 Sep;178[3 Pt 1]:814-8. [DOI: 10.1016/j.juro.2007.05.040].
12. Ashley RA, Inman BA, Sebo TJ, Leibovich BC, Blute ML, Kwon ED, Zincke H. Urachal carcinoma: clinicopathologic features and long-term outcomes of an aggressive malignancy. Cancer. 2006 Aug 15;107[4]:712-20. [DOI: 10.1002/cncr.22060].

13. Mostofi FK, Thomson RV, Dean AL. Mucous adenocarcinoma of the urinary bladder. Cancer. 1955; 8:741-758. [DOI: 10.1002/1097-0142[1955]8:4<741:aidcncr2820080417>3.0.c0;2-c].

14. Sheldon CA, Clayman RV, Gonzalez R, Williams RD, Fraley EE. Malignant urachal lesions. J Urol. 1984 Jan;131[1]:1-8. [DOI: 10.1016/s0022-5347[17]50167-6].

15. Dhillon J, Liang Y, Kamat AM, Siefker-Radtke A, Dinney CP, Czerniak B, Guo CC. Urachal carcinoma: a pathologic and clinical study of 46 cases. Hum Pathol. 2015 Dec;46[12]:1808-14. [DOI: 10.1016/j.humpath. 2015. 07. 021].

16. Reis H, Szarvas T. Urachal cancer-current concepts of a rare cancer. Pathologe. 2019 Jun;40[Suppl 1]:31-39. English. [DOI: 10.1007/s00292-018-0516-9].

17. Schubert GE, Pavkovic MB, Bethke-Bedürftig BA. Tubular urachal remnants in adult bladders. J Urol. 1982 Jan;127[1]:40-2. [DOI: 10.1016/s0022-5347[17]53595-8].

18. Paner GP, Lopez-Beltran A, Sirohi D, Amin MB. Updates in the Pathologic Diagnosis and Classification of Epithelial Neoplasms of Urachal Origin. Adv Anat Pathol. 2016 Mar;23[2]:71-83. [DOI: 10.1097/PAP.0000000000000110. PMID: 26849813].

19. Gopalan A, Sharp DS, Fine SW, Tickoo SK, Herr HW, Reuter VE, Olgac S. Urachal carcinoma: a clinicpathologic analysis of 24 cases with outcome correlation. Am J Surg Pathol. 2009 May;33[5]:659-68. [DOI: 10.1097/PAS.0b013e31819aa4ae].

20. Schmitt W, Baptista M, Ferreira M, Gomes A, Germano A. Urachal Adenocarcinoma: A Case Report with Key Imaging Findings and Radiologic-Pathologic Correlation. Case Rep Radiol. 2018 Feb 28; 2018: 4935261. [DOI: 10.1155/2018/4935261]. 